Relations industrielles

Industrial Relations

\title{
Inside a Sensitivity Training Groups, by T.R. Weschler \& J. Reisel, Institute of Industrial Relations. University of California, Los Angeles, Monogra Series: 4, 134 pp.
}

\section{C.-R. Giroux}

Volume 15, numéro 2, avril 1960

URI : https://id.erudit.org/iderudit/1022048ar

DOI : https://doi.org/10.7202/1022048ar

Aller au sommaire du numéro

Éditeur(s)

Département des relations industrielles de l’Université Laval

ISSN

0034-379X (imprimé)

1703-8138 (numérique)

Découvrir la revue

Citer ce compte rendu

Giroux, C.-R. (1960). Compte rendu de [Inside a Sensitivity Training Groups, by

T.R. Weschler \& J. Reisel, Institute of Industrial Relations. University of

California, Los Angeles, Monogra Series: 4, 134 pp.] Relations industrielles /

Industrial Relations, 15(2), 285-286. https://doi.org/10.7202/1022048ar

Tous droits réservés (C Département des relations industrielles de l’Université Laval, 1960
Ce document est protégé par la loi sur le droit d'auteur. L'utilisation des services d'Érudit (y compris la reproduction) est assujettie à sa politique d'utilisation que vous pouvez consulter en ligne.

https://apropos.erudit.org/fr/usagers/politique-dutilisation/ 
de recherche très importants. 1)L'école des relations humaines pourra étudier les motivations des représentants syndicaux. Toutefois, elle doit accepter comme inévitables les conflits entre le capital et le travail. De plus, elle ne doit plus avoir comme philosophie l'asservissement du travail aux seuls buts du capital. 2) Pour étudier la structure du travail et ses relations avec la capital, il serait bon de consulter les documents officiels tels que les constitutions, les chartes, les contrats et de découvrir comment ils ont évoluer. 3) Le contrôle exercé par les 《leaders 》 syndicaux reflète-t-il le fonctionnement d'une institution démocratique? Une grande quantité d'informations serait obtenue par une observation systématique des relations entre le meneur et ses subalternes. 4) Quels sont les moyens de communications développés ou utilisés par les représentants syndicaux? 5) Quels sont les conflits d'autorité causés par la fondation de groupes nationaux? Dans quelle mesure $\mathrm{la}$ bureaucratie influence-t-elle l'administration du syndicat? 6) Il y a très peu de recherche sur les problèmes administratifs rencontrés par le représentant syndical et sur la suffisance des procédures administratives. 7) Un autre domaine très dynamique consisterait à évaluer et à modifier les perceptions réciproques du capital et du travail.

Cet ouvrage de MacDonald résume et commente très bien la recherche sur l'autorité syndicale. Concis et bien étoffé, ce volume se doit d'intéresser les chercheurs dans le domaine des sciences humaines et sociales.

\section{C.-R. Giroux}

Inside a Sensitivity Training Groups, by T.R. Weschler \& J. Reisel, Institute of Industrial Relations. University of California, Los Angeles, Monogra Series: $4,134 \mathrm{pp}$.

Un drame humain, un drame social, un drame authentique, puisqu'il s'agit de l'impuissance d'un groupe à solutionner ses problèmes. Le groupe ne réussit même pas à s'entendre sur sa structure et sur un mode d'opération. Ce petit ouvrage est des plus captivants. Le lecteur suit l'évolution du groupe, ses tentatives de croissance, ses capitulations face aux conflits émotifs et aux attitudes négatives de certains membres. Le lecteur attend vainement un dénouernent de dernière heure. Le groupe capitule enfin, laissant le lecteur aux prises avec un profond sentiment de mélancolie. La croissance des membres a-t-elle avortée!

Le présent ouvrage décrit comment tout membre d'un groupe doit non seulement se comprendre objectivement, mais accepter ses propres déficiences avant de pouvoir entretenir des interrelations efficaces avec ses compagnons. Pendant longtemps l'on tenta de développer et d'entraîner les administrateurs et les gérants en leur enseignant les méthodes administratives, en augmentant leurs connaissances techniques, pour enfin se rendre à l'évidence que les résultats attendus étaient loin de se concrétiser parce que les individus se montraient incapables d'entretenir les contacts humains flexibles et exempts de conflits. La croissance et le développement d'un individu ne peut s'opérer qu'en fonction de ses contacts humains. Dans les cas extrêmes il faut avoir recours à une thérapie de groupe. Pourquoi ? Parce que c'est la seule façon de calibrer les réactions individuelles à un diapason commun, i.e. en fonction d'un comportement commun.

Plusieurs méthodes ont été mises au point pour provoquer la croissance de l'individu au sein du groupe. Trois méthodes sont surtout connues, celle du «Sensitivity Training - University of California », celle du \& Leaderless Group Discussion - Louisiana State University » et celle du «National Training Laboratory ». Les trois méthodes utilisent la technique non-directive de Rogers.

Le comportement du groupe se manifeste par les interactions entre les membres. Ces échanges individuels oscillent entre deux pôles, la subjectivité des intérêts et des problèmes personnels, et l'objectivité engendrée par la compréhension et l'acceptation des réactions des autres. Si chacun des membres du groupe réussit à se dégager de ses préoccupations personnelles, à comprendre et à accepter ses besoins, ses craintes et ses tendances, il se montrera alors plus réceptif envers ses camarades. plus compréhensif et enfin plus conciliant. Cette croissance individuelle limitera les comportements irrationnels des membres. 
Alors le groupe pourra commencer son oeuvre de créativité en essayant d'atteindre des objectifs communs.

Pour que le comportement de l'individu et du groupe soit modifié par un entraînement commun, Welschler et Reisel assument ce qui suit: a) Les participants trouveront en eux-mêmes les sources essentielles de leur propre croissance. Un entraînement de groupe force l'individu à analyser de façon objective. Si l'individu n'aime pas ses attitudes et son comportement, à lui d'initier les changements nécessaires. L'entraînement permet à l'individu de s'observer dans une nouvelle perspective. b) En général l'homme veut contrôler sa propre destinée. Il veut que ses contacts sociaux soient exempts de craintes, de doutes et d'attitudes négatives. c) Un entraînement social efficace se base sur l'interaction des membres. Chacun considère les autres comme des miroirs qui reflètent ses attitudes, ses valeurs sociales, son comportement. L'instructeur encourage les participants à communiquer entre eux le plus possible, à exprimer ouvertement leurs attitudes et à interpréter ce qui se passe. d) Un entraînement de groupe permet au membre de comparer son comportement à celui du groupe. Il a également l'opportunité de se familiariser avec des attitudes et des idées nouvelles. e)Enfin, la croissance personnelle du participant est grandement favorisée par une situation qui lui assure le respect de ses droits et de son individualité.

Qu'arrive-t-il si les membres du groupe ne réussissent pas à initier une action commune, s'ils ne s'entendent pas sur les buts à atteindre, s'ils sont plus préoccupés de leurs intérêts personnels que des sentiments des autres? Le présent ouvrage en est un exemple. A ce moment-là, le groupe régresse totalement et la seule solution en est une d'autorité: alors, le «leader» doit prendre la direction et imposer ses décisions.

\section{C.-R. Grroux}

What Every Supervisor Should Know,

by Leslie R. Bittel. McGraw Hill Book, New York, 1959, 451 pp.

Le contremaître est une figure dramatique dans toute entreprise commerciale et industrielle. En effet, il doit sans cesse réconcilier les intérêts divers de ses supérieurs, de ses confrères, de ses employés, des représentants du syndicat, etc. Aussi, le contremaître n'a pas le temps d'analyser sa position, et encore moins les théories sociales et économiques qui caractérisent sa fonction. $\mathbf{C e}$ qu'il veut ce sont des réponses et des conseils concrets à des problèmes réels.

Trop nombreuses sont les publications qui vantent telle technique de travail ou telle méthode de supervision, car souvent le contremaître n'a même pas le temps ni le désir de lire ces bouquins parce qu'ils n'apportent rien de neuf. Enfin, Bittel a préparé pour le contremaître un ouvrage qui deviendra pour plusieurs un livre de chevet. Au delà de 700 problèmes y sont traités sous forme de paragraphes courts. Chacun répond à une question claire et spécifique. Le style est clair, concis, dénudé de tout artifice et surtout exempt de verbiage. Malgré tout, le texte est logique et consistant. Il en résulte un volume dont la lecture est facile et rapide.

Les problèmes $y$ sont traités de façon réaliste et ils intéresseront tous ceux qui occupent des postes de commande. L'auteur répond à deux questions par page. Ce qui permet au lecteur de se retrouver rapidement lorsqu'il a quelques moments de disponibles, On y trouve des problèmes qui ont rarement été abordés ailleurs, tels que la supervision des femmes, l'art de donner des ordres, la supervision des employés âgés et tout un chapitre sur le développenent personnel. Il est étonnant de constater avec quelle facilité il explique certains principes psychologiques de l'apprentissage. Son chapitre sur la pensée créatrice sera révélateur pour plusieurs, En effet, Bittel soutient que tous les gens doués d'une intelligence moyenne peuvent apprendre à penser et à découvrir des idées nouvelles. Il suffit de s'y mettre systématiquement.

L'auteur sait très bien qu'il ne résout pas tous les problèmes qu'il aborde, aussi n'hésite-t-il pas à donner une foule de références. Il est plutôt rare de trouver un ouvrage de vulgarisation qui se termine par une bibliographie de six pages. C'est un ouvrage qui aidera et orientera tous ceux qui s'occupent d'entraînement et de formation des cadres.

C.-R. Gmoux 